\title{
Procesos de migración y familias transnacionales de la Sierra Queretana, México
}

\author{
Alejandra Elizabeth Urbiola Solis \\ Universidad Autónoma de Querétaro \\ Alejandra.urbiola@uaq.mx
}

Resumen: La Sierra Queretana, en el centro de México, incluye dos municipios: Amealco de Bonfil y Huimilpan; en ambos, la migración hacia los Estados Unidos es un fenómeno frecuente que ha modificado paulatinamente la forma de organización de las familias. El análisis de la migración se realiza tomando en cuenta al grupo familiar y los cambios en la división sexo-genérica del trabajo y los efectos económicos y culturales que conlleva. El artículo expone las condiciones de vida de los grupos en familias transnacionales, en particular de las mujeres y los parientes que no migran; el desarrollo de emprendimientos y empresas familiares como alternativas posibles del uso de remesas. La pregunta de investigación que se planteó fue conocer las estrategias de supervivencia de las familias transnacionales en relación con los aspectos de cuidado, compromiso y reciprocidad. La metodología es de orientación cualitativa, hermenéutica y de fenomenología; se incluyó una revisión documental y se realizaron entrevistas semiestructuradas. Como técnicas para la triangulación, se contrastaron resultados de entrevistas y se utilizó la observación no participante. El trabajo de campo se realizó durante los veranos de 2018 y 2019. Se realizaron diecisiete entrevistas a miembros de familias transnacionales y cinco a funcionarios de los municipios estudiados, de la oficina nacional de empleo y una organización civil de apoyo al migrante.

Entre los principales resultados se encuentra que las mujeres que no migran enfrentan nuevas condiciones económicas que les implican romper con los esquemas tradicionales de género, aunque la construcción subjetiva de un nuevo rol de género es paulatina, no se asume de manera inmediata; muchas mujeres que no migran no logran romper los roles asignados en la esfera privada ya que las nuevas condiciones de vida que enfrentan no las desligan de su condición de género en la familia, pero al mismo tiempo enfrentan responsabilidades como jefas de familia. Se percibe como una doble jornada laboral que sin embargo no las enfrenta con los miembros de la familia extensa. Los emprendimientos llevados a cabo involucran estrategias de negocios en red que son posibles gracias a las familias extensas y a los vinculos entre los 
migrantes. El éxito o fracaso de los mismos es percibido no solo como una consecuencia del mercado sino también en relación con la posibilidad de asegurar el envío de remesas.

Palabras clave: migración (090106), división sexo-genérica del trabajo (090202), cambio cultural (100201).

\section{Migration processes and transnational families of the Sierra Queretana, Mexico}

Abstract: The Queretana mountain range in central Mexico includes two municipalities: Amealco de Bonfil and Huimilpan. In both, migration to the United States is a frequent phenomenon that has gradually modified the way families are organized. The analysis of migration takes into account the family group and the changes in the gender-generic division of labor and the economic and cultural effects that it entails. The article exposes the living conditions of groups in transnational families, particularly women and non-migrant relatives; the development of enterprises and family businesses as possible alternatives to the use of remittances. The research sought to determine the survival strategies of transnational families in relation to aspects of care, commitment and reciprocity. The methodology is qualitative, hermeneutical and phenomenological. A documentary review was included and semistructured interviews were conducted. As techniques for triangulation, interview results were contrasted and non-participant observation was used. The fieldwork was carried out during the summers of 2018 and 2019. 17 interviews were conducted with members of transnational families and 5 with officials from the municipalities studied, the national employment office and a civil organization supporting migrants.

Among the main results is that women, who do not migrate, face new economic conditions that involve them breaking with traditional gender schemes, although the subjective construction of a new gender role is gradual. Many women who do not migrate fail to break the roles assigned in the private sphere because the new living conditions they face do not separate them from their gender status in the family. However, at the same time, they face responsibilities as heads of their families. They are perceived as carrying out a double working day which nevertheless does not bring them into conflict with members of the extended family. The ventures carried out involve business network strategies that are possible thanks to extended families and links between migrants. Their success or failure is perceived not only as a consequence of the market but also in relation to the possibility of sending remittances.

Keywords: migration (090106); sex-generic division of labor (090202); cultural change (100201). 


\section{Planteamiento del problema}

La historia migratoria entre México y Estados Unidos data del siglo xIx. Durante los años previos a la guerra con Estados Unidos (1846-1848), la relación entre los dos países fue muy tensa debido a la independencia de Texas y su anexión posterior a los estados de la Unión; posteriormente, ya restablecida la paz, el proceso de migración quedó bien establecido, primero, como una migración circular, para, posteriormente, incluir casos de migración definitiva. De acuerdo con Massey, Durand y Malone (2009), existen seis etapas en la historia de la migración con Estados Unidos; esta clasificación también ha sido retomada por otros autores como Alarcón (2011). A continuación, se listan las principales características que enuncian los autores por etapa:

a) Período de enganche, 1900-1920. Consistió en un sistema de contratación derivado de la demanda creciente de mano de obra barata para la construcción de obras como el ferrocarril y las cosechas en el campo. Esta etapa se corresponde con la migración circular basada en la forma de contratación en el lugar de origen de los migrantes mexicanos y con contratos predeterminados por tiempo y lugar. Para Massey, Durand y Malone (2009), esta etapa corresponde a una condición laboral de trabajo forzado. En el caso de Alarcón (2011), el primer período se relaciona con los cambios en la oferta de trabajo en condición migrante y la demanda en los sectores de la minería, agricultura e industria en los Estados Unidos. Retoma el proceso desde finales del siglo xviII, donde existían prohibiciones para los trabajadores de origen chino y japonés en los Estados Unidos. Los trabajadores mexicanos tuvieron formas de contratación legal desde finales del siglo xix hasta 1917 con el cambio en la legislación de migración con una base restrictiva en función del origen (Gamio, 1991).

b) Deportaciones, 1921-1941. Este período se relaciona con elementos del contexto mundial y específicamente norteamericano, como fue la crisis de 1929. Durante el proceso de contracción económica, la pérdida de empleos para los norteamericanos se tradujo en xenofobia para los trabajadores mexicanos, así como en medidas de control migratorio con un sistema de cuotas y deportaciones masivas. En este período la fuerza laboral estaba en un circuito migratorio circular y era posible el control a través de la Patrulla Fronteriza (creada en 1924).

c) Programa Bracero, 1942-1964. Durante este período las deportaciones comenzaron a disminuir al tiempo que comenzaban a darse más permisos a mano de obra mexicana como braceros en el campo (1942-1964) y en las vías de ferrocarril (1942-1945). El contexto en el que se encontraba 
la economía norteamericana fue en una primera etapa el de la Segunda Guerra Mundial y en una segunda etapa la guerra de Corea, así como en la Guerra Fría; en política interna, existían dos fuerzas, una, procuraba mayor cantidad de visados para braceros, que, sin embargo, recibían un ingreso bajo comparativamente con los trabajadores nativos, mientras la otra parte basaba su rechazo en un discurso nacionalista y de igualdad en las condiciones laborales y de salarios.

d) Indocumentados, 1965-1986. Al terminar el Programa Braceros, la condición migratoria de migrantes europeos y asiáticos cambió. Comenzó un sistema de preferencias con topes a la migración por continente y cuotas de visados a partir de 1968. Se aprobó la IRCA, Inmigration Reform and Control Act. El cambio se debe a la modificación en la estructura ocupacional en los Estados Unidos una vez terminada la guerra de Vietnam (1975-1975) y la presión por mejorar las condiciones de los trabajadores en territorio norteamericano, así como a la lucha por los derechos civiles. La migración durante el período era circular, nunca cesó, pero se volvió ilegal e invisible. De acuerdo con Alarcón (2011), los migrantes provenían de regiones del centro de México y se desplazaban por períodos de tiempo durante un año hacia los Estados Unidos para ocuparse en trabajos agrícolas fundamentalmente. Al no existir una restricción mayor, ya en 1980 había doce millones de trabajadores en condición migratoria irregular.

f) Fase bipolar, 1987-2007. Durante este período que incluye el último cuarto del siglo $\mathrm{xx}$ y principios del siglo $\mathrm{xxI}$, las condiciones de la economía mexicana habían cambiado; al comenzar un proceso de desregulación económica y apertura comercial, se buscó estimular la demanda agregada y el crecimiento del producto interno bruto a través de las exportaciones netas y la inversión privada. Sin embargo, la adopción del modelo neoliberal en México tuvo como parteaguas la crisis económica de 1892 y, posteriormente, la de 1994. En el caso mexicano, las condiciones laborales no fueron buenas: bajos salarios o salarios con topes debido a la política de «pactos» entre los decisores económicos, impulsada por el Gobierno desde 1982-1988, y, posteriormente, la apertura comercial y la especialización de la economía mexicana en productos de maquila, autopartes y productos de exportación agrícola incluyeron la firma del Acuerdo General sobre Aranceles y Comercio (GATT) y el Tratado de Libre Comercio con América del Norte. En los Estados Unidos, el Programa Silva, vigente entre 1977 y 1981, apoyó la entrada de trabajadores migratorios en condición regular. Aunque, derivada de las condiciones domésticas, la mi- 
gración en condición irregular comenzó a aumentar y entre 1965 y 1986 entraron 25 millones de mexicanos de forma ilegal (Massey et alii, 2009). Alarcón (2011), por su parte, afirma que durante el período y hasta 2004 había once millones de trabajadores migrantes en condición irregular. Este éxodo masivo fue consecuencia de las crisis económicas en México y el diferencial salarial con la economía norteamericana. Asimismo, la migración comenzó a modificar sus condiciones, y pasó de tener una característica circular a ser en muchos casos definitiva. Muchos migrantes comenzaron a ver la posibilidad de encontrar mejores condiciones de vida para ellos y para sus familias en los Estados Unidos y se incorporaron como ciudadanos (Durand, 2016).

+ g) Fase actual, 2007 en adelante. En este período comenzaron a surgir discursos ambivalentes en ambos lados de la frontera. Por un lado, en México, se habló de un cambio hacia la «democracia» y un mayor apoyo a los empresarios micro y pequeños desde el año 2000. El énfasis en el apoyo a la inversión privada atraería mayores empleos, ingresos y consumo, y cerraría un círculo virtuoso que se traduciría en crecimiento del producto interno bruto; sin embargo, la falta de oportunidades, el empobrecimiento y exclusión social de grandes grupos de la población incrementaron los volúmenes de migrantes hacia los Estados Unidos. Por otra parte, la reivindicación de los derechos humanos y condiciones laborales, así como el concepto de «trabajo digno», repercutiría en ambos países, pero sería mayor en los Estados Unidos. Los cambios en la legislación migratoria van de acuerdo con las condiciones de los mercados y las demandas políticas de los grupos dentro de Estados Unidos (Bustamante, 1997). La legislación migratoria se ha vuelto más estricta con vigilancia y penalización para el migrante no solo por razones estrictamente económicas, sino también por las condiciones sociales en la región: incremento del narcotráfico y problemas sociales en Centroamérica.

En este orden de ideas, puede decirse que la relación binacional México-Estados Unidos sigue siendo difícil en relación con el tema migratorio. Las remesas que se envían hacia México se han utilizado para financiar el crecimiento y puede afirmarse que complementan los flujos de financiamiento externo privado y no crean obligaciones de deuda. Sin embargo, constituyen la evidencia de una integración elevada entre ambas economías, lo que implica que no pueden convertirse en mecanismos de salida a la crisis económica cuando en Estados Unidos se desacelera el crecimiento del producto interno bruto. 
Aunada a esta dependencia o integración entre ambas economías, a nivel doméstico, en la región de estudio, constituyen un mecanismo que solventa la falta de crédito y con ello es posible tener empresas familiares; asimismo, con apoyo gubernamental en el programa $3 \mathrm{X} 1$ se arman proyectos productivos en coparticipación con los grupos de migrantes organizados en los Estados Unidos y los Gobiernos federal y municipales en cada Estado. El gasto de las remesas no solo se destina a emprendimientos económicos, ya que en muchas ocasiones su uso es complementario o para proyectos específicos: de infraestructura social, de servicios comunitarios, educativos y productivos. Estos últimos en coparticipación con el Gobierno y en diferentes porcentajes.

familias extensas con miembros que comenzaron a quedarse en los Estados Unidos. Por otra parte, muchos migrantes en la región de Amealco de Bonfil y Huimilpan han construido redes de amigos y migrantes, además de las que pudieran tener dentro de la familia en la región y en los Estados Unidos para mejorar sus condiciones de vida.

\section{Fundamentación teórica sobre la migración}

Las teorías que se ocupan de explicar las causas que subyacen al proceso migratorio son varias y pueden dividirse en dos grandes grupos: las que tienen como base la explicación neoclásica sobre la elección racional, la medición de los costos y beneficios esperados de la migración y la dotación de factores de producción capital y trabajo, y aquellas que buscan explicar la migración en función de otras variables no económicas, ya sean estas internas al sujeto y a la familia y a la condición social o al contexto en el que vive el sujeto. Este segundo grupo busca una explicación interdisciplinar, más holística, y no solamente económica, al proceso migratorio.

\subsection{Características generales de los modelos neoclásicos}

El campo disciplinar de los modelos es la economía, la mayoría de ellos (Arango, 2003) fueron desarrollados durante el período de 1960-1970 (Ranis y Fei, 1961; Harris y Todaro, 1961; Sjaastad, 1962); se refieren al sujeto que está en condición de migrar o que ya ha tomado la decisión y se encuentra de forma regular o irregular. Se acepta el supuesto de «elección racional» y del homo economicus que es capaz de calcular hasta cierto punto los costos de oportunidad de la decisión de migrar; otros factores que incluyen estas teorías son el diferencial en la asignación de recursos: factores trabajo y capital que se traduce en abundancia de mano de obra y escasez de la misma en las economías más desarrolladas. La decisión de migrar es una 
decisión de costo-beneficio ya sea del individuo o del grupo al que este pertenece (Stark, 1982, citado en Arango, 2003), e incluye un coeficiente de riesgo, un cálculo basado en la distancia y la inversión de capital humano que supone el proceso. A diferencia de los primeros modelos neoclásicos de Ranis y Fei (1961) y Todaro (1961), esta explicación sobre la migración considera a la familia como una unidad donde es posible diversificar estrategias para tener fuentes de ingreso alternativas. Esta diferencia - familia versus individuo - y la búsqueda de la diversificación de ingresos y no la maximización de los mismos constituyen un paso importante en las explicaciones teóricas sobre el fenómeno migratorio al introducir factores sociales entre las causas principales. Arango $(2003,2007)$ se refiere al conjunto de modelos neoclásicos como modelos push-pull, por las fuerzas de atracción y expulsión de ambas economías, donde el diferencial en los precios relativos de los factores de producción y las determinantes estructurales determinan la expulsión de mano de obra (Zlotnik, 1992). Las principales críticas al conjunto de modelos es que no explican las tasas de migración dispares en países con condiciones similares, es decir, en condiciones de desigualdad en la distribución de renta y asignación de factores de producción. Tampoco explican por qué bajo esas condiciones, la mayoría de las personas no migran, no explican la migración diferencial. Por otro lado, tampoco incluyen la dimensión política o los determinantes culturales y sociales en las decisiones sobre migración que realiza un sujeto o un grupo. Sin embargo, se considera que son útiles para explicar las migraciones internas.

\subsection{Características de las teorías sociológicas de la migración}

Este grupo incluye teorías sobre los grupos familiares y las estrategias de migración de las unidades domésticas, así como de las condiciones laborales en mercados de trabajo duales y segmentados. Corresponden a este grupo la teoría o proposiciones de rango intermedio de la nueva economía de las migraciones y la teoría de la segmentación del mercado de trabajo propuesta inicialmente por Piore (1969, 1973, 1975, citado por Fernández-Huerga, 2000), conocida también como teoría de los mercados duales o segmentados, la teoría de la dependencia o del sistema mundial y la teoría del capital social, sistemas migratorios y causación acumulativa. Retomando los trabajos de la corriente neoclásica, se incluyen en este grupo otras causas de migración diferentes de la necesidad económica o nivel individual o la búsqueda de igualdad en la retribución a los factores de producción (oferta diferencial), para incluir los siguientes elementos:

a) Privación relativa más que pobreza absoluta.

b) Demanda permanente estructural de trabajadores inmigrantes en economías industriales. 
c) Desempleo estructural y exclusión social.

d) Mejora en las condiciones de vida de miembros de la familia migrante o familia transnacional.

Este grupo de teorías incluye elementos políticos, sociales y culturales o simbólicos para explicar la migración, sin determinar todavía cuál es el peso de cada uno de los factores en el sistema migratorio. La decisión puede incluir a un individuo o a una familia, pero, a diferencia de los modelos neoclásicos, el sujeto está «anclado» en un contexto geográfico-temporal y sujeto a normas culturales que tiende a perpetuar como migrante. Se incluyen elementos como el capital social y se enfoca en explicaciones no solo «micro», sino «meso» $y$ «macro» al analizar la vinculación del migrante en su origen y destino con redes de apoyo que pueden ser de carácter familiar y personal, pero también de empleo y vinculación con otros migrantes. En su parte «macro» establece un análisis de los sistemas migratorios, con flujos de entrada y salida, además de la explicación sistémica de países en «centro-periferia». La teoría propuesta por Massey (2015) establece que la migración es un fenómeno autosostenido y perpetrador y que existen factores y mecanismos que facilitan el proceso de autoperpetración: privación relativa, cultura de emigración, distribución perversa del capital humano y estigma sobre el trabajo de migrantes. Asimismo, el conjunto de teorías incluye elementos que permiten comprender el proceso de migración desde el sujeto y el grupo, pero al mismo tiempo implican complejizar el análisis, ya que el sujeto tiene particularidades: condición de género, identidad étnica y religiosa y grupo etario (Urbiola y Ortiz, 2019).

\subsection{La familia transnacional}

Durante el proceso de migración, el sujeto que deja el hogar lo hace por razones muy variadas, siendo el deseo de mejorar su condición de vida o circunstancia económica muy importante en su decisión. La familia o grupo de apoyo puede ser nuclear, pero en la gran mayoría de los casos es extensa y puede o no guardar relación de parentesco con el migrante; adquiere la condición de transnacional porque el movimiento implica «redes entre los e/in-migrantes en sus destinos y sus contrapartes en origen que tienen las siguientes características: regularidad, sostenibilidad y simultaneidad» (Molina, 2019). Las principales características de la familia en condición transnacional son las siguientes: ausencia de corresidencia, dispersión espacial, condición multilocal, sentido de colectividad, intercambios a distancia, interdependencia relacional y sentido de copresencia, virtual y simbólico (Ariza, 2012). Los migrantes establecen lazos en un espacio o campo social transnacional o donde desde un país de origen establecen acuerdos de rec- 
iprocidad y compromiso al interior de la familia (Ariza, 2013; Ariza y Portes, 2007; Herrera, 2008; Jacques, 2008).

Cualquier miembro de la familia sin distinción de género puede insertarse en un circuito migratorio; para el caso de México, durante el período de 1965 a 1986 la migración irregular aumentó (Massey et alii, 2009), y en las dos etapas subsiguientes a partir de 1987 (Alarcón, 2011) aumentó el número de mujeres y niños migrantes. A partir del cambio en los mercados laborales la condición de reproducción tradicional de los roles asignados por género en el interior de la familia también comenzó a modificarse. Esta condición se observó con anterioridad en grupos de familias extensas relacionadas con mercados de trabajo locales y/o regionales. El proceso de industrialización de la región centro de México implicó un crecimiento de municipios y regiones con nuevas áreas de producción industrial; el desarrollo de parques industriales cerca de los municipios colindantes a los de la Sierra Queretana modificó las estrategias de subsistencia de las unidades domésticas campesinas (Urbiola, 1996) en los municipios más industrializados y comenzaron procesos de migración interna condicionados por los cambios en los mercados laborales. La reorientación de la demanda obedeció a las necesidades industriales; las familias extensas dedicadas a actividades agropecuarias diversificaron sus estrategias de ingreso y se incorporaron como asalariados a la industria local (Carrillo, 2017; Urbiola, 2017).

El género como categoría analítica resulta una herramienta útil para mostrar el cambio en las estrategias de reproducción familiar en grupos de migrantes, y en el caso aquí presentado, de miembros de familias transnacionales. Alejada de los principios esencialistas y biologistas sobre los roles «naturales» para hombres y mujeres, la teoría de género nos permite plantear la importancia de la construcción social de los procesos migratorios (Burin y Meler, 2009 y 2010). Nos muestra que las mujeres, a diferencia de los hombres, en los procesos migratorios están expuestas a una doble vulnerabilidad, la primera en relación con las categorías impuestas socialmente a lo largo del tiempo, y la segunda, por la condición de edad y discriminación del grupo migrante, en particular cuando se trata de minorías o grupos étnicos (Chodorow, 1984; Palacios, 2016). En el caso de la Sierra Queretana existe un proceso de transición en los roles de género de migración masculina hacia femenina a partir de los perfiles de demanda laboral, coexistiendo con roles asignados por tradición y una condición de sexo genérica vinculante al proceso migratorio (De Barbieri, 1993; Rubin, 1986). 


\section{El diseño y la metodología}

La metodología que se siguió fue la cualitativa; se consideró que debido al carácter empírico de la investigación y al enfoque sobre los procesos sociales que involucra la migración, desde la construcción subjetiva de los migrantes, el principio de idoneidad (Flick, 2015) apuntaba a una metodología cualitativa como la mejor estrategia. A través de la investigación, se buscó mostrar las condiciones de los migrantes en familias transnacionales; la pregunta de investigación que se planteó fue conocer las estrategias de supervivencia de las familias transnacionales en relación con los aspectos de cuidado, compromiso y reciprocidad.

Son numerosos los trabajos sobre las condiciones de migración, pero en el artículo se busca mostrar que el proceso migratorio es vinculante hacia los miembros de la familia. El trabajo de campo se realizó en dos fases; en una primera fase fue necesaria la revisión documental y estadística de los municipios de la Sierra Queretana para conocer indicadores económicos de contexto y estructurales en la región; en una segunda fase, se hizo necesario establecer una «sintonía»o «rapport» (Galeano, 2018) para formar el método y la forma más apropiados para el muestreo. A través del mapeo se consigue identificar una realidad sociocultural anclada a un espacio físico con una temporalidad y actores determinados (Galeano, 2018).

En esta investigación el enfoque a resaltar es el punto de vista de los sujetos, desde la fenomenología (Creswell, 1998, 2013); se utilizó un muestreo en bola de nieve para realizar las entrevistas a sujetos, hombres y mujeres en familias extensas con las características que Ariza (2012) describe para familias transnacionales: ausencia de corresidencia, dispersión espacial en los municipios y los Estados Unidos; multilocalidad y un sentimiento colectivo de bienestar y unidad.

Para la selección de informantes se utilizaron tres criterios: la pertenencia a la familia, el conocimiento del proceso investigado y la experiencia como migrante. El trabajo de campo se realizó durante los veranos de 2018 y 2019. Se llevaron a cabo 17 entrevistas a personas de familias transnacionales con miembros en procesos migratorios regulares o no regulares. La información de las entrevistas se analizó considerando las configuraciones de cuidado intergeneracional, en familias de hasta tres generaciones, donde la migración es un fenómeno cotidiano (Creswell, 2013). Las otras dimensiones de análisis fueron las que se refieren a los compromisos de los miembros de la familia y a la reciprocidad. Estas se objetivan no solo en los cuidados de los menores sino también en los emprendimientos y decisiones financieras que se toman en relación con las remesas; por último, la configuración sexo-genérica del trabajo y los cambios que ocurren en la organización del núcleo familiar. Se utilizaron como técnicas para la triangulación de la información la observación no participante y cinco entrevistas a personas 
relacionadas con el proceso de migración: funcionarios de los municipios y de la oficina nacional de empleo, así como de una organización civil de apoyo al migrante. La validez de la información se realizó a través de la contrastación de las explicaciones que proporcionaron los miembros de las familias, los funcionarios municipales y los expertos sobre el tema de migración a nivel general en el municipio y en relación con las familias en los dos municipios. Asimismo, se verificó que no existieran casos nulos o marginales que pudieran considerarse "atípicos» a la situación observada en la región (Giménez, 2012).

En relación con el alcance y la extrapolación de los resultados obtenidos en la Sierra Queretana, se considera que la comparación con otras poblaciones no estudiadas en municipios colindantes es posible si se mantienen condiciones socioeconómicas y culturales similares en su inserción en el circuito migratorio México-Estados Unidos.

En un inicio la condición de exclusión y pobreza en los dos municipios es paradójica, si se considera que al mismo tiempo ambos colindan con los dos municipios más ricos del estado de Querétaro. Podría pensarse que el crecimiento de los municipios de Querétaro, El Marqués, Corregidora y San Juan del Río podría atraer a gran parte de la población; asimismo, los incrementos en años de instrucción les posibilitarían una mejor condición laboral. Sin embargo, es paradójico, porque, a pesar de la cercanía a los parques industriales, la reorientación económica del territorio en esos municipios no ha tenido el impacto esperado y no se puede decir que ha ayudado a mejorar sustancialmente las condiciones de vida y/o bienestar de la población en esos municipios (el recorrido entre Querétaro y Huimilpan es de 37 km, y entre Querétaro y Amealco de Bonfil es de 75,6 km). A partir de lo anterior, el objetivo de la investigación fue conocer las condiciones de vida de los grupos en familias transnacionales, en particular de las mujeres y los parientes que no migran. Desde el inicio se observó que muchos habitantes de las cabeceras municipales pensaban que era mejor migrar a los Estados Unidos que ir a trabajar hacia la ciudad de Querétaro o San Juan del Río.

El análisis de la migración que se propone en esta investigación no se realiza desde los modelos neoclásicos econométricos, sino desde la familia transnacional, considerando que es en la unidad donde se toman decisiones de gasto, de ahorro y de inversión de un grupo (Román, 2013), y donde es posible el inicio de emprendimientos o empresas familiares.

Es importante aclarar que, debido a la condición migratoria no regular de muchos migrantes, la posibilidad de grabar todas las entrevistas se desvaneció. El miedo a ser descubiertos y poner en riesgo las redes de apoyo informales para los migrantes fueron las causas principales. Dada la condición transnacional de las familias, se mantienen redes activas en ambos países, y, en muchos casos, un 
migrante puede cambiar su condición migratoria de regular a irregular en un período de tiempo corto; en otras ocasiones, aunque algunos miembros de una familia pueden migrar por un período de tiempo corto y con visa de trabajo desde México, otros miembros dentro de la misma familia pueden preferir hacerlo a través de un intermediario (coyote); en otras ocasiones, se establecen diferentes mecanismos para la migración de las esposas y los hijos. Algunos menores también carecen de papeles que acrediten la nacionalidad, ya sea mexicana o norteamericana, al haber nacido de mujeres mexicanas en condición migratoria no regular. El temor a las deportaciones y a perder el contacto con la familia en ese tipo de situación les previene sobre el tipo de información que pueden compartir.

\section{La región de estudio}

Amealco de Bonfil y Huimilpan pertenecen al estado de Querétaro, este es una de las entidades donde existe alta intensidad migratoria hacia los Estados Unidos $^{1}$. La entidad está dividida en cinco regiones: Sierra Gorda, Semidesierto Queretano, Valles Centrales, Bajío Queretano y Sierra Queretana, agrupando 18 municipios (ver tabla 1). Los municipios con mayor migración se encuentran en la Sierra Gorda (5), el Semidesierto Queretano (1) y la Sierra Queretana (2).

Tabla 1. Regiones y Municipios del estado de Querétaro

\begin{tabular}{|c|c|c|c|c|c|}
\hline & Municipios & Número & $\begin{array}{l}\text { Grado de Margina- } \\
\text { ción (INEGI, 2010) }\end{array}$ & Alto & $\begin{array}{l}\text { Muy } \\
\text { alto }\end{array}$ \\
\hline Sierra Gorda & $\begin{array}{l}\text { Arrollo Seco, Jalpan de } \\
\text { Serra, Pinal de Amoles, } \\
\text { San Joaquín, Landa de } \\
\text { Matamoros }\end{array}$ & 5 & Alto y muy alto & 2 & 3 \\
\hline $\begin{array}{c}\text { Semidesierto } \\
\text { Queretano }\end{array}$ & $\begin{array}{l}\text { Peñamiller, Tolimán, Co- } \\
\text { lón, Cadeyta de Montes }\end{array}$ & 4 & Medio & & \\
\hline Valles Centrales & $\begin{array}{c}\text { El Marqués, Ezequiel } \\
\text { Montes, Tequisquiapan, } \\
\text { Pedro Escobedo, San } \\
\text { Juan del Río }\end{array}$ & 5 & Bajo y muy bajo & & \\
\hline Bajío Queretano & Querétaro, Corregidora & 2 & Muy bajo & & \\
\hline $\begin{array}{l}\text { Sierra Quere- } \\
\text { tana }\end{array}$ & $\begin{array}{c}\text { Huimilpan, Amealco de } \\
\text { Bonfil }\end{array}$ & 2 & Alto y medio & 1 & \\
\hline
\end{tabular}

Fuente: elaboración propia con datos de INEGI (2000; 2010). Cuéntame. Población. Migración.

1 Querétaro es un estado que se ubica en la región de México conocida como El Bajío y que incluye a los estados con mayor migración hacia los Estados Unidos: Guanajuato, Jalisco y Michoacán de Ocampo, y a otros dos estados del centro de la República: Estado de México y Puebla (INEGI, 2010). 
El fenómeno migratorio se ha acentuado desde mediados del siglo $\mathrm{xx}^{2} \mathrm{y}$ tiene un origen multicausal: pobreza y exclusión social, falta de empleo en la región, pocas oportunidades de emprendimiento y apoyo gubernamental insuficiente. Los programas de apoyo vigentes surgieron en 2002 con el Programa Iniciativa Ciudadana $3 \mathrm{X} 1$; actualmente el programa $3 \mathrm{X} 1$ para migrantes busca apoyar iniciativas de ciudadanos organizados en los estados con mayor índice migratorio. Sin embargo, a pesar de este programa y los de apoyo consular, no se ha podido solucionar de fondo el problema de rezago social de muchas comunidades. Existen en forma paralela otros problemas: al tener el estado de Querétaro contigüidad con los estados de Guanajuato y Michoacán, se ha registrado un incremento de la inseguridad debido al narcotráfico y al robo de combustibles de la empresa gubernamental Pemex.

En sus inicios, la migración comprendida durante el período del Programa Bracero (1942-1964) se corresponde con una migración de tipo circular con puestos de ocupación en el sector primario; posteriormente, el flujo migratorio obedeció también a las condiciones de los municipios y de las familias extensas con miembros que comenzaron a quedarse en los Estados Unidos. Por otra parte, muchos migrantes en la región de Amealco de Bonfil y Huimilpan han construido redes de amigos y migrantes, además de las que pudieran tener dentro de la familia en la región y en los Estados Unidos para mejorar sus condiciones de vida (Plan Municipal de Desarrollo de Amealco, 2016; Plan Municipal de Desarrollo de Huimilpan, 2015).

\section{Los principales resultados}

De acuerdo con Ariza (2012), las familias transnacionales presentan las siguientes características:

- Ausencia de corresidencia o copresencia.

+ Dispersión espacial.

+ Multilocalidad.

- Sentimiento colectivo de bienestar y unidad.

En el grupo de personas entrevistadas se encontró que las familias a las que ellos pertenecían reunían las características anteriores; que muchas de esas familias son extensas de hasta tres generaciones y que el tiempo de permanencia de cada sujeto en Estados Unidos varía en relación con las necesidades económicas del grupo familiar, pero también está influido por el sentimiento colectivo de lo que significa el bien común familiar y las expectativas sobre el futuro que pueden

2 El fenómeno en México tiene raíces históricas anteriores, de mediados del siglo xix. 
tener en los Estados Unidos, específicamente sobre lo que Massey (en Arango, 2003; 2017) denomina causación acumulativa. En la mayoría de las personas entrevistadas se encontró que al menos un miembro de la familia había migrado o estaba migrando y que por lo general el cuidado de los hijos y parientes mayores lo hacían las mujeres. Aunque existe un arraigo profundo por la tierra natal, muchas mujeres (madres y abuelas) consideran que, en Estados Unidos, los hijos pueden crecer mejor, con mejores oportunidades. A partir de esa creencia, algunas mujeres expresan la posibilidad de estadías largas o permanencia definitiva de los miembros de sus familias en los Estados Unidos, aunque ello implique una responsabilidad permanente para ellas.

A través de la revisión bibliográfica, la observación en campo y las entrevistas realizadas se presentan los resultados obtenidos en relación con las configuraciones de cuidado en familias que pueden identificarse como transnacionales; en relación con los compromisos y expectativas de cuidado y los aspectos relacionados con los emprendimientos y decisiones financieras. Se identificaron dos componentes característicos de la región Sierra Queretana:

- Una identidad étnica (otomí) y religiosa (católica) en el caso del municipio de Amealco de Bonfil; el municipio tiene una historia fincada en relación con el grupo indígena otomí (hñähñú), desde el período colonial mexicano (1521-1810). Esa identidad se expresa en el idioma, el sincretismo religioso, y el calendario ritual anual que mezcla ambas culturas.

- Interdependencia relacional en familias extensas; cada uno de los miembros de la familia extensa es importante. Los abuelos apoyan a los hijos, cuidan a los nietos mientras los padres están en movimiento.

Se encontró evidencia que confirma que los sujetos mantienen intercambios a distancia, en cierto sentido existe interdependencia relacional y un sentido de copresencia que puede ser física en algunos casos, pero que es mayormente virtual y simbólica, como afirma Ariza (2012). El acceso a los medios de comunicación y transporte ha permitido una comunicación más frecuente e intensa entre los migrantes y sus familias.

Por otra parte, se reconoce que la mayoría de los migrantes son hombres. La diferencia porcentual entre hombres y mujeres en México para 2015 (INEGI) es de 2\%; $51 \%$ para mujeres y $49 \%$ para hombres sobre el total de la población; en el caso de Amealco de Bonfil, la diferencia es de 7\% para 2005 y 2010 y de 9\% para 2015 (ver gráfico 1). En el caso de Huimilpan, el diferencial entre hombres y mujeres es menor que el de Amealco de Bonfil, pero mayor que el nacional: de 3,5 y $9 \%$, respectivamente (gráfico 2). El registro de migración para Huimilpan en el período 2005-2010 se triplicó. 
Gráfica 1. Población de hombres y mujeres en Amealco de Bonfil.

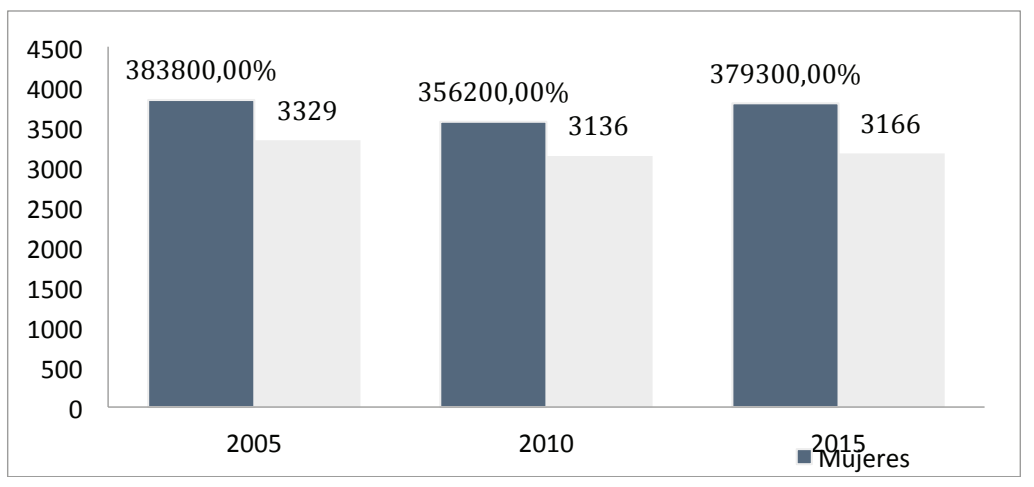

Fuente: elaboración propia con datos de INEGI. Amealco de Bonfil 2005-2015. Encuesta de población por municipio.

\section{Gráfica 2. Población de hombres y mujeres en Huimilpan}

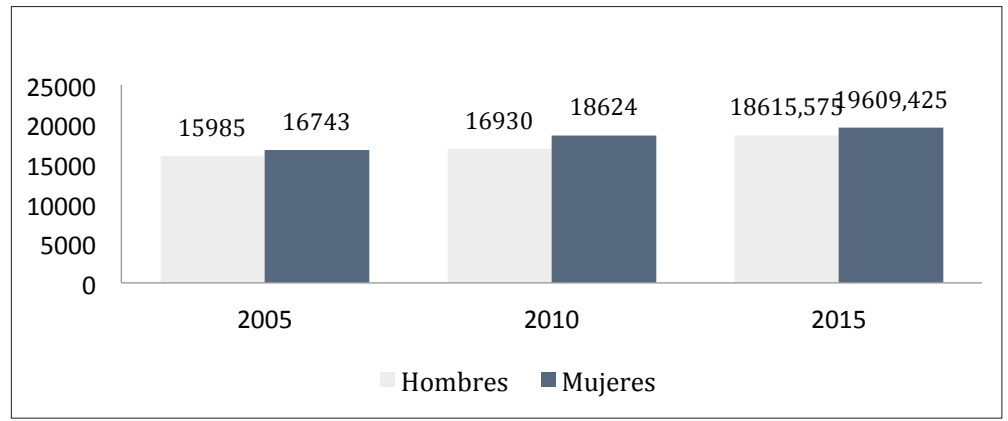

Fuente: elaboración propia con datos de INEGI. Huimilpan 2005-2015. Encuesta de población por municipio

Se encontró que muchas mujeres son cabeza de familia en la localidad, ellas desempeñan el rol de jefe de familia frente a las autoridades municipales y escolares, pero es el esposo quien toma las decisiones sobre los gastos de la casa y los hijos, aunque se encuentre en los Estados Unidos confirmando que la independencia de muchas mujeres no significa la autonomía económica, como lo define Coria (2008).

La primera vez fue siete años, después tres años, y así, y esta última vez estuve allá cinco años, cinco años y meses... Mis hijos se los encargaba a una hermana... Sí, sí, les mandé [dinero] con cuidado, no les mandé de más porque les dije que yo no quería que se hicieran parejeras con la demás gente, solo como para ir comiendo y pa' una necesidad, siempre había por ahí un guardadito, pero no le di 
a mi familia acceso, que diga exceso, de dinero, ¿̇vedá?, le di el necesario, no, porque se hace la gente parejera (E3, hombre migrante retornado, jefe de familia).

Desde el 95 y 96, del 95 al 2000 iba y venía, 2000, 2001 iba y venía cada año, cada año, pero a partir de esa fecha ya se puso más difícil ir y venir porque él todo el tiempo fue inmigrante, nunca ha tenido la oportunidad de visa y ahorita ya del 2000 a la fecha ha ido tres veces, de las cuales ha durado entre cinco o seis años... [El dinero] ha sido de gran ayuda (E4, hija de migrante).

El rol tradicional de la mujer en el desempeño de las actividades de maternaje y cuidado del hogar sigue vigente, pero se agrega el de gestora frente a las oficinas gubernamentales para la obtención de apoyos a la producción del campo si la tenencia de la tierra es ejidal y apoyos para actividades empresariales de emprendimiento, así como la de fungir como cabeza de familia frente a las autoridades escolares en el caso de los hijos menores.

Mi esposa siguió su rutina como de matrimonio normal, sabes que yo me voy allá y tú te quedas aquí a cuidar a los muchachos, pero si es el cambio como de comida y de vestir (E1, hombre migrante, jefe de familia).

Yo me quedo a cuidar a mis hijos (E6, esposa de migrante, jefa de familia).

$\mathrm{Al}$ irse ellos una de madre es la que se queda a cargo de los hijos porque el papá sale a trabajar lejos (E7, esposa de migrante retornado).

Pues los primeros años sí me acogí con mi familia y ya después de que mis hijos crecieron ya me quedé en mi propia casa (E10, esposa y hermana de migrante, jefa de familia).

En relación con los compromisos y expectativas de cuidado, la mayoría de las mujeres incorpora al trabajo del cuidado de los menores a las abuelas; el hecho de compartir espacios de terreno grandes les permite tener varias viviendas dentro de un mismo solar y compartir las responsabilidades de cuidado de los menores y los ancianos y las responsabilidades económicas. Las principales configuraciones de cuidado fueron las siguientes: abuela-madre presentes y padre a distancia; abuela-abuelo-tíos presentes y madre y padre a distancia; abuelaabuelo presentes y madre a distancia. Aunque no es tan común, en Amealco de Bonfil y Huimilpan se comienza a percibir la migración de menores y mujeres como una oportunidad para la familia. Esta investigación no profundizó sobre las causas del fenómeno, sin embargo, apuntan a una mayor educación (años de 
instrucción), oportunidades laborales específicas por género y reducción en el número de hijos en parejas estables.

Las mujeres migrantes pueden tener «hijos en destino», término que Ariza (2012) utiliza para denominar a los hijos nacidos en el lugar de destino como resultado de una decisión individual o sin que exista una planeación de por medio. Dependiendo de las políticas migratorias, los proyectos para reagrupar a las familias se postergan y la maternidad/paternidad a distancia se complica utilizando a los padres/abuelos/tíos para la educación y el cuidado de los hijos. De ahí que las remesas sean muy importantes para la supervivencia del núcleo familiar tanto en dinero como en especie. Existe evidencia que demuestra que dentro de una misma familia una mujer, esposa, hija o hermana puede tener a su esposo, a sus hijos o hermanos en procesos de migración. Esta condición implica estrategias en familias extensas sobre el apoyo para la crianza y los compromisos con los adultos mayores.

Con respecto al uso del dinero de remesas, varios informantes indicaron que las remesas funcionan para financiar los emprendimientos, solventando la falta de crédito doméstico. En muchas ocasiones, los miembros de las familias no son considerados sujetos de crédito por la banca comercial. En su mayoría, el destino es la inversión en la vivienda, mejorar la infraestructura de la casa o el equipamiento. Un segundo rubro mencionado fue el de la educación y cuidado de los hijos: pago de colegiaturas y de servicios médicos particulares, sobre todo cuando las mujeres van a dar a luz o para el tratamiento médico de los ancianos. Existe también un tipo de remesa en especie porque cada año algunos migrantes visitan las comunidades y traen consigo objetos que sus familias pueden utilizar y/o vender en la comunidad.

Las remesas constituyen una fuente de financiamiento de la demanda en ambos municipios, ya que a pesar de que existe un cambio notable en la orientación productiva de los otros municipios colindantes, la región estudiada mantiene un acentuado estancamiento de las actividades productivas la mayor parte de la superficie se dedica a las actividades agropecuarias, por otra parte, el 12,4\% de la población en Huimilpan se encuentra en pobreza extrema, es decir, con al menos tres carencias sociales: carencia alimentaria, condición de vivienda no adecuada y falta de servicios de drenaje, luz y agua, además de condiciones laborales precarias; en el caso de Amealco de Bonfil la población en esta condición es del 25,4\% del total registrado (Plan Municipal, 2015).

Los emprendimientos realizados son microempresas familiares, en su mayoría de grupos extensos; aunque se encontró evidencia de un caso en el municipio de Huimilpan, en la comunidad de San Pedro Norte, de un grupo conformado 
por nueve familias que abrieron una empresa de servicio turístico con apoyo gubernamental y ahorros de remesas y que ha sido exitosa durante dieciséis años (Maldonado, Pérez y Ávila, 2017).

Con respecto a los programas gubernamentales de movilidad laboral se encontró que, a través del Servicio Nacional de Empleo (SNE), existen actualmente personas beneficiadas que han tenido o tienen todavía la condición de migrante, ya sea regular o en condición irregular. En relación con los programas, estos son para trabajadores agrícolas temporales hacia Estados Unidos y se incluye ahora también a Canadá (ver tabla 2).

Tabla 2. Programa Repatriados Trabajando 2012-2018.

\begin{tabular}{|c|c|c|c|c|c|c|c|}
\hline MUNICIPIO & 2012 & 2013 & 2014 & 2015 & 2016 & 2017 & 2018 \\
\hline ACULCO, EDO DE MEX & & 2 & & & & & \\
\hline AMEALCO DE BONFIL & 5 & 9 & 4 & 1 & 3 & 2 & 3 \\
\hline CADEREYTA DE MONTES & & & 3 & & & & 2 \\
\hline EPITACIO HUERTA, MICH & & 1 & 1 & & 1 & 1 & \\
\hline EZEQUIEL MONTES & 2 & & 1 & & & & 1 \\
\hline HUICHAPAN, HGO & & & 1 & & & & \\
\hline PEDRO ESCOBEDO & & & 1 & & & 1 & \\
\hline PEÑAMILLER & & 1 & 1 & & & & \\
\hline PINAL DE AMOLES & & & & & & & 2 \\
\hline POLOTITLÁN, EDO.MEX & & 1 & & & & & \\
\hline QUERÉTARO & & & & & & 1 & \\
\hline SAN JOAQUÍN & & & & & & 1 & \\
\hline SAN JUAN DEL RÍO & 25 & 14 & 23 & 6 & 21 & 12 & 21 \\
\hline TEQUISQUIPAN & 3 & & 3 & 1 & 1 & & 2 \\
\hline TOTALES & 35 & 28 & 38 & 8 & 26 & 18 & 31 \\
\hline
\end{tabular}

Fuente: Servicio Nacional de Empleo. Unidad Regional San Juan del Río.

Existe una demanda importante de trabajadores no calificados en Estados Unidos; en general, la mano de obra se orienta al sector de la construcción; a nivel operativo, en el sector manufacturero, y en el sector de servicios, en el turismo. Existe una precarización en los mercados laborales en el país de destino, aunque los salarios fueron reportados por los entrevistados como más altos en comparación con los que reciben en los dos municipios. La forma de ingreso al mercado laboral internacional no siempre es legal ni a través del programa de Servicio Nacional de Empleo, pero la mayoría de los entrevistados conoce la forma de inserción no regular a través de un coyote.

En relación con los beneficiados de programas y que hayan sido trabajadores migrantes, se encontró que se atendió a 27 repatriados desde 2012 hasta 2018 en Amealco de Bonfil. Para el caso de Huimilpan, no se encontró evidencia de atención a través del SNE. En cuanto a los trabajadores en Canadá se encontró que la institución tiene registro de 329 migrantes temporales para actividades agropecuarias procedentes de Amealco de Bonfil para el período de 2011-2018. En 
una entrevista con la funcionaria del SNE, comentó que las personas interesadas en trabajar en Canadá deben cumplir ciertos requisitos que se consideran indispensables antes de recibir la aprobación (visa) para trabajar: conocimiento de las actividades de cultivo de frutas, cereales, tabaco, árboles y pasto, así como de horticultura, apicultura y actividades pecuarias. Sin embargo, también comentó que existen requisitos extra no relacionados con la actividad, como el de no tener tatuajes en partes visibles del cuerpo y el vivir en zonas rurales del país.

En el caso de los programas de apoyo directo a la comunidad, el programa 3X1 es considerado prioritario para las autoridades del municipio de Huimilpan, debido a la importancia de la contribución económica de los migrantes al municipio. En este programa, la participación de los migrantes es del $25 \%$ y el monto de apoyo federal puede ser hasta de 50.000 dólares para los proyectos de infraestructura social y de servicio comunitario; los otros proyectos que se apoyan son el educativo y el productivo, que tienen como objeto apoyar a las familias, ya sea en comunidad, con al menos diez familias en un proyecto común, o por unidad doméstica, en varias comunidades del municipio; en este caso pueden ser de tres a cinco con familias. Sin embargo, las personas entrevistadas del municipio de Huimilpan no tenían conocimiento sobre cómo funciona el programa o qué tipo de apoyo proporciona.

\section{Conclusiones}

La migración constituye una actividad importante en los municipios de Amealco de Bonfil y Huimilpan en la región de la Sierra Queretana. La migración es un proceso en el que se involucran no solo el sujeto migrante sino también la familia. Aquellos miembros de familias en condición transnacional mantienen un sentimiento colectivo de bienestar a pesar de no existir una copresencia física constante o permanente. Las familias extensas dividen el trabajo y el cuidado de sus integrantes por género; se encontró que la mayoría de los sujetos migrantes eran hombres y que, en general, la migración es una salida para la precariedad y la exclusión social a pesar de que la región ha tenido un cambio económico notable. Aunque existen explicaciones teóricas sobre el origen de la migración que refieren a los aspectos económicos o de dotación de factores de producción, la migración en ambos municipios incluye también causas socioculturales referidas a las expectativas económicas y de oportunidades en los Estados Unidos y a las estrategias familiares de subsistencia.

La migración va acompañada de cambios en los roles de género, las mujeres se enfrentan a nuevas condiciones económicas que les implican romper con los esquemas tradicionales, aunque, en muchas ocasiones, la construcción subjetiva 
de un nuevo rol de género es paulatina. Esta nueva condición no se asume de manera inmediata; muchas mujeres que no migran no logran romper los roles asignados en la esfera privada, y al mismo tiempo, se enfrentan con una responsabilidad nueva como jefes de familia. Se percibe como una doble jornada laboral que, sin embargo, no las enfrenta con los miembros de la familia extensa. Las configuraciones de cuidado más comunes fueron las de abuela-madre presentes y padre a distancia; abuela-abuelo-tíos presentes y madre y padre a distancia, y abuela-abuelo presentes y madre a distancia.

Los emprendimientos llevados a cabo involucran estrategias de negocios en red que son posibles gracias a las familias extensas y al vínculo con los migrantes, aunque la mayoría del dinero que entra al hogar por remesas se gasta en el sostenimiento de la familia, en la construcción y en el equipamiento del hogar. Existe también el envío de remesas en especie.

En ambos municipios se encontró una cultura del migrante, es decir, formas específicas para el traslado y búsqueda de empleo, redes de apoyo en ambos países, estrategias de comunicación, así como reconocimiento en ambos municipios de la derrama económica relacionada con la migración. El Gobierno municipal ha buscado reconocer y apoyar al migrante, aunque los entrevistados no sabían cómo funcionaban los programas de apoyo. 


\section{Referencias}

Alarcón, Rafael (2011).“U.S. Immigration Policy and the Mobility of Mexicans (1882-2005)". Migraciones internacionales, 6(1), 185-218.

Arango, Joaquín (2003). "La explicación teórica de las migraciones: Luz y sombra". Migración y Desarrollo, Red Internacional de Migración y Desarrollo, (1), $1-30$.

Arango, Joaquín (2007)."Las migraciones internacionales en un mundo globalizado". Vanguardia Dossier, (22), 6-15.

Ariza, Marina (2012). "Vida familiar transnacional en inmigrantes de México y República Dominicana en dos contextos de recepción”. Revista de Estudios Transfronterizos, XII(1), 17-47.

Ariza, Marina (2013). Inmigración y trabajo reproductivo. El servicio doméstico en Estados Unidos en tiempos de globalización. México: Universidad Autónoma de México.

Ariza, Marina y Portes, Alejandro (2007). El país transnacional. Migración mexicana y cambio social a través de la frontera. México: Universidad Nacional Autónoma de México. Instituto de Investigaciones Sociales.

Burin, Mabel y Meler, Irene (2009). Varones. Género y subjetividad masculina. Buenos Aires: Librería de las Mujeres.

Burin, Mabel y Meler, Irene (2010). Género y familia. Poder, amor y sexualidad en la construcción de la subjetividad. Buenos Aires: Paidós.

Bustamante, Jorge (1997). Cruzar la linea: la migración de México a los Estados Unidos. México: Fondo de Cultura Económica.

Carrillo, Marco Antonio (2017). Expresiones del trabajo en Querétaro. México: CONCYTEQ, UMEST.

Coria, Clara (2008). El sexo oculto del dinero. Formas de la dependencia femenina. Buenos Aires: Paidós.

Creswell, John W. (1998). Qualitative Inquiry and Research Design. Choosing among Five traditions. Thousand Oaks, California: SAGE.

Creswell, John W. (2013). Qualitative Inquiry and Research Design. Choosing among Five Approaches (3. ${ }^{\text {a }}$ ed.). Thousand Oaks, California: SAGE.

Chодоrow, Nancy (1984). El ejercicio de la maternidad psicoanálisis y sociología de la maternidad y paternidad en la crianza de los hijos. Barcelona: Gedisa.

De Barbieri, Martha Teresita (1993). "Sobre la categoría género. Una introducción teórico metodológica”. Debates en Sociología, (18), 145-169.

Durand, Jorge (2016). "Procesos y patrones migratorios México-Estados Unidos". Conferencia Magistral. El Colegio de la Frontera Norte. 
Fernández-Huerga, Eduardo (2010)."La teoría de la segmentación del mercado de trabajo: enfoques, situación actual y perspectivas de futuro". Investigación Económica, LXIX(273), 115-150. Disponible en: <http://www.scielo. org.mx/pdf/ineco/v69n273/v69n273a4.pdf>.

Flick, Uwe (2015). El diseño de investigación cualitativa. Madrid: Morata.

Galeano, María Eumelia (2018). Diseño de proyectos en la investigación cualitativa. Colombia: Fondo Editorial Universidad EAFIT.

Gamio, Manuel (1991)."Número, procedencia y distribución de los emigrantes mexicanos en los Estados Unidos2. En: Jorge Durand (coord.). Migración México Estados Unidos Años Veinte. México: Dirección General de Publicaciones del Consejo Nacional para la Cultura y las Artes.

Giménez, Gilberto (2012). "El problema de la generalización en los estudios de caso". Cultura y Representaciones Sociales, 7(13), 40-62.

Gobierno Municipal de Amealco de Bonfil (2016). "Plan Municipal de Desarrollo Amealco de Bonfil, Querétaro". México: Gaceta oficial del Gobierno Municipal de Amealco de Bonfil, Querétaro. Disponible en: <file://C:/Users/ Alejandra\%20U/Desktop/DOCTOS\%20OFICIALES\%20AMEALCO\%20Y\%20HUIMILPAN/planes\%20municipales/ANEXO\%20GACETA\%209\%20(PLAN\%20MUNICIPAL\%20DE\%20DESARROLLO\%20).pdf>.

Gobierno Municipal de Huimilpan (ca. 2015)."Plan Municipal de Desarrollo Huimilpan 2015-2018". Querétaro: <file://C:/Users/Alejandra\%20U/ Desktop/DOCTOS\%20OFICIALES\%20AMEALCO\%20Y\%20HUIMILPAN/planes\%20municipales/plan\%20municipal-huimilpan.pdf $>$.

Herrera, Gioconda (2008). "Políticas migratorias y familias transnacionales: migración ecuatoriana en España y Estados Unidos”. En: Gioconda Herrera y Jacques Ramírez (coords.). América Latina migrante: estado, familias, identidades. Quito: FLACSO; Sede Ecuador: Ministerio de Cultura del Ecuador.

Instituto Nacional de Estadística Geografía e Informática. "Censos de Población y Vivienda 2000 y 2010". INEGI. México.

Instituto Nacional de Estadística Geografía e Informática. "Base de Datos de Migración de los municipios del estado de Querétaro, 1990, 2000, 2005 y 2010". INEGI. México.

Instituto Nacional de Estadística Geografía e Informática (2000). “Amealco de Bonfil"” Cuaderno Estadístico Municipal 2000. INEGI. México.

Instituto Nacional de Estadística Geografía e Informática (2014). “Anuario estadístico y geográfico de Querétaro." 2010. INEGI. México. 
Instituto Nacional de Estadística Geografía e Informática (2010). "Huimilpan. Informe anual de la educación 2010". INEGI. México.

Instituto Nacional de Estadística Geografía e Informática (2015). "Huimilpan. Resultados de la encuesta intercensal 2015". INEGI. México.

Jacques, Ramírez (ed.) (2008). América Latina migrante: Estado, familias, identidades. Ecuador: Ministerio de Cultura del Ecuador, Flacso, Ecuador.

Maldonado, Candelaria; Pérez, Felipe y Ávila, Marisol (2017). “Diagnóstico Participativo de la Sociedad Cooperativa del Campamento Ecoturístico, San Pedro Huimilpan, Querétaro y la Toma de Decisiones como Micro Empresa Familiar". Cuadernos de Investigación Turística y Economía, pp. 95104. Recuperado el 30 de septiembre del 2018, de <https://es.calameo.com/ $\mathrm{read} / 00366135780 \mathrm{baa} 30 \mathrm{fe} 2 \mathrm{fc}>$.

Massey, Douglas (2017). Especialidad en Migración Internacional. Evolución del Sistema Migratorio Norteamericano. Seminario virtual llevado a cabo en el Colegio de la Frontera Norte, Colef.

Massey, Douglas; Durand, Jorge y Malone, J. Nolan (2009). Detrás de la trama. Políticas migratorias entre México y Estados Unidos. Miguel Ángel Porrúa; Universidad Autónoma de Zacatecas, México.

Massey, Douglas (2015). "A missing element in migration theories". Migration Letters, 12(3), 279-299.

Molina, José Luis. "Conceptos básicos de transnacionalismo". [En línea] [29 de marzo de 2019]. Disponible en <http://pagines.uab.cat/joseluismolina/sites/pagines.uab.cat.joseluismolina/files/Conceptos\%20b\%C3\%A1sicos\%20 de\%20transnacionalismo.pdf $>$.

Palacios, Yennesit (2016). "Perspectiva de género en los fenómenos migratorios: estudio desde Europa y América Latina”. Revista CES Derecho, 7(2), 145-162.

Piore, Michael Joseph (1969). "On-the-job training in dual labor markets". En: A. Weber, F. Cassell y G. Woodrow (coords.) (1969). Public-private Manpower Policies. USA: Madison, Industrial Relations Research Association.

- (1973)."The importance of human capital theory to labor economics. A dissenting view". Documento para la Twenty-Sixth Annual Winter Meeting, Industrial Relations Research Association. USA: Madison

- (1975). "Notes for a theory of labor market stratification". En: R. EdwArds, M. Reich y D. Gordon (coords.) (1975). Labor Market Segmentation. Lexington, DC: Heath and Co.

Rubin, Gayle (1986). "El tráfico de mujeres: notas sobre la «economía política» del sexo". Revista Nueva Antropología, VIII(30), 95-145. 
Román, Patricia (2013). "La familia como unidad de análisis en el estudio de la migración". En: Eduardo Sandoval (coord.). Familia y migración. México: Editorial Porrúa.

Urbiola, Alejandra y Ortiz, Ricardo (2019). "Migración internacional, una constante en la relación binacional México-Estados Unidos". Revista CIMEXUS, XIV(1), 103-119. DOI: <https://doi.org/10.33110/cimexus140105>.

Urbiola, Alejandra (1996). La diversificación en las estrategias tradicionales de subsistencia de las unidades domésticas de Los Cues, Querétaro (tesis de Antropología Social). Distrito Federal: Escuela Nacional de Antropología e Historia.

Urbiola, Alejandra (2017). “Reorientación económica en Querétaro. Hacia un diagnóstico organizacional." En: Marco CArrillo (coord.). Expresiones del trabajo en Querétaro. México: CONCYTEQ, UMEST.

Zlotnik, Hania (1992). Empirical identification of international migration systems. Oxford: Clarendon Press. 